\title{
Image Segmentation with Neurocomputers
}

\author{
G.L. Bilbro \\ Mark White \\ and \\ Wesley Snyder
}

Center for Communications and Signal Processing Electrical and Computer Engineering Department

North Carolina State University

August 1987

CCSP-TR-87/12 


\section{Introduction}

Our ultimate application interest is the automated understanding of images, especially non-biological images such as range or synthetic aperture radar. In this report, we describe one aspect of the processing of such images, segmentation, and show that one can design a neural network to perform this computation. An important step ${ }^{14}$ in image analysis is segmentation. An image is an array of discrete sampled values called pixels. An image is understood when the objects portrayed in that image are recognized ${ }^{3}$ or at least characterized. An object is characterized in terms of the segments of the image that it subtends. An image segment ${ }^{1,6}$ is a collection of pixels that satisfies certain conditions of adjacency and similarity.

Image segmentation has been usefully viewed as a connected component analysis which can be done very quickly ${ }^{19,5}$ using associative memory once the connectedness of pixels is known. Connectedness subsumes the two conditions of adjacency and similarity.

Conventional measures of connectedness for computation on serial machines depend on estimates of partial derivatives ${ }^{16,9,4}$ of the image function. These derivatives are estimated by convolving the image with a kernel that has to be large because derivatives are notoriously sensitive to noise. Usually it is also necessary to fix the shape of the kernel because the additional computational expense of dynamically adapting a large kernel to the data is unacceptable. But when such a large rigid kernel straddles two distinct regions, it blurs their properties. This results in boundaries that are so wide that they must be thinned $d^{4}$, or so broken by gaps that they must be closed ${ }^{17,18}$. Closing and thinning are typically ad hoc and of ten sub-optimal.

\subsection{Design of a Neural Network for Segmentation}

It is easy to construct a Lyapunov ${ }^{10}$ or energy function $E$ and therefore a Hopfield ${ }^{13,11}$ or Boltzmann ${ }^{2,7}$ neural model which will compute an optimal (or near optimal) segmentation in the precise sense of minimizing $E$. We have simulated such a Boltzmann segmenter with encouraging experimental results that are briefly summarized in the appendix of this document. In the remainder of this section, we will discuss the theoretical definition of this problem, the design considerations of the neural network, and some generalizations of the consequent segmentation algorithm.

Consider first the problem of optimally segmenting a one dimensional image of possibly noisy pixels (such as a profile or a raster line of a range or luminance image). We take the image to have the form

$$
\{x, z=f(x)\}_{x=1}^{x=N} \text {. }
$$

Optimality is defined in terms of the energy

$$
E_{\text {tot }},
$$

which is a function of the joint state of $S N$ neurons 


$$
\left\{V_{s}(x)\right\}_{s=1 \quad x=1}^{s=S} x=N
$$

In a Boltzmann model, each $V_{s}(x)$ can assume either the value of 0 or 1 . In the case of two segments, we set $S=2$, and say that when $V_{1}(x)=1$ and $V_{2}(x)=0$ the pixel at $x$ is in segment $s=1$. If the state values are reversed, then the pixel belongs to segment 2 . Other state values do not correspond to physical assignments.

In the elaboration of $E_{t o t}$, we will specify a minimum feature size $r$ for segments in order to specify the tradeoff between segment resolution and noise tolerance. We must also define similarity precisely in terms of some analytic function to which we require each segment conform. The simplest function is the constant function; it results in segments optimally described as plateaus. We will choose a linear model which leads to optimal straight line segments. We could have chosen a quadratic model to allow smoothly curving segments. Finally, we have some freedom in the definition of the adjacency of pixels. The set of pixels that interact with the pixel $x$ defines the neighborhood of $x$. We arrange our neural net to have the same topography as the original image so that adjacent pixels are represented by adjacent neurons. We assume no a priori knowledge about the location of features in the image, and will define the neighborhood of any $x$ in terms of a fixed set of displacements, $\{\nu\}$. For consistency with the previous specification of a minimum feature size $r$, we must choose $\{v\}$ to have a radius on the order of $r$. We can reduce the number of interconnects and therefore the hardware complexity by using the sampling theorem to determine the sparsest acceptable set $\{v\}$ necessary to reproduce the image signal: We take the set $\{\nu\}$ to be uniform with spacing on order of $r$.

We now construct an energy function over the joint state of our $2 N$ neurons (that is, over all segmentations) which will be minimized by the desired segmentation. We will find three contributions to this energy. We will need an exclusion term to prevent a pixel from being assigned to more than one segment. We will use a cohesion term to suppress small segments. And we use the image to construct a consistency ${ }^{8}$ term that penalizes the assignment of a pair of nearby but dissimilar pixels to the same segment.

The exclusion term

$$
E_{\text {excl }}=\sum_{x=1}^{N} V_{3=1}(x) V_{s=2}(x)
$$

includes a contribution from each pixel that penalizes non-physical configurations with pixels belonging to both segments. In the Hopfield model ${ }^{11}$, where the state of the neuron varies continuously in $[0,1]$, we would have to modify the exclusion term somewhat. The exclusion term limits the physically acceptable solution state space as a subset of the computational state space.

The cohesion term is a sum over adjacent pixels

$$
E_{\text {coh }}=-\sum_{z=1}^{N} \sum_{s=1}^{S} V_{j}(x) V_{s}(x+1)
$$

that favors configurations in which adjacent pixels have the same label. 
In our current model, the actual image is represented in the consistency term

$$
E_{\text {cons }}=\sum_{x=1}^{x=N} \sum_{\{v\}} \sum_{s=1}^{s=S} V_{s}(x) V_{s}(x+v) P(x, x+v) \text {, }
$$

where the penalty function is

$$
P(x, y)=\int_{x}^{y}|f(t)-M(x, t, y)| d t .
$$

The term in absolute value is the discrepancy of the actual pixel value at $t$ from its value as predicted by the model $M(x, t, y)$ based on the values at $x$ and $y$. In our linear case, the model

$$
M(x, t, y)=\frac{f(x)(y-t)-f(y)(t-x)}{y-x},
$$

is the usual linear interpolation formula. $P(x, y)$ can be understood as the total discrepancy of the actual values of pixels in the interval $[x, y]$ from their predicted values. This penalty $P(x, y)$ only contributes to $E_{\text {cons }}$ when the pixels at $x$ and $y$ are in the same segment: when $V_{s}(x)=V_{s}(y)=1$ for some $s$.

Now the total energy for the network is

$$
E_{\text {tot }}=A E_{\text {excl }}+B E_{\text {coh }}+C E_{\text {cons }} \text {. }
$$

The values of the three coefficients $A, B$ and $C$ are difficult to predict theoretically. However we have experimentally determined that the performance of our network is not very sensitive to the values of these weights. It appears that simulated annealing makes the Boltzmann net tolerant to a wide range of values. The final low temperature stable state is robust to variation of these coefficients. We believe that the final high gain stable state of the analogous Hopfield model will perform similarly if the search for final states is pursued from the low gain limit.

It should also be noted that a degenerate state

$$
\left\{V_{s}(x)=0\right\}_{s=1 \quad s=1}^{s=S} x=N
$$

exists for an unbiased network strictly governed by the energy equations derived here. This state is avoided by biasing all neurons toward the " 1 " state in a fashion analogous to that described by others ${ }^{12}$.

\subsubsection{Using the Neural Network in a Segmentation Algorithm}

We have used the standard simulated annealing procedure ${ }^{15}$ to study the operation of this neural network as it constructs a segmentation from various input images. The appendix contains an example of simulation results from a pilot study of segmentation in 1-dimensional space. 


\subsubsection{Generalization to Several Segments in Two Dimensions}

Although we have called this a segmentation algorithm for two segments, in fact it will label any number of segments alternately with 1 and 2 whenever they satisfy the other conditions of optimality as discussed previously. It is easy to apply this algorithm to multi-segment images by regarding its output as a coloring of the image rather than a segmentation. In fact, it is a 2-coloring, but since any linear map is 2-colorable, our two segment model is sufficient. If we now say that two pixels are connected if they are adjacent and if they have the same color, we can complete the segmentation with the connected component analysis discussed above ${ }^{19}$.

The segmentation of two dimensional images is formally similar to the one dimensional case. The elements of the minimal interconnection neighborhood $N$ are related to cylindrical Bessel functions, but are data independent constants used to reduce the hardware complexity at design time in the eventual VLSI fabrication. It is known that any planar graph is 4 -colorable and this suggests that $S=4$ is appropriate for the general two dimensional case. However, it appears that the errors incurred by a $S=3$ system may be tolerable. In any case, we have not yet investigated the tradeoffs between coloring errors and the complexity of networks with larger $S$.

\section{Appendix}

Here we illustrate the simulation of a neural network designed to segment data in 1dimensional space. The diagram below illustrates a 4 neuron section of a larger 64-neuron network. This figure illustrates only one of the network configurations that can be used for segmentation. Each row of neurons represents a different segment. In this example, a two segment classifier is implemented. Each neuron within a row represents a different pixel, located at a different position along the $x$ dimension. Each neuron in a column represents a different segment but the same pixel and $x$ position. There are three types of interconnections between the neurons. The respective weights (ie. synaptic transmission coefficients) of each type of interconnection are labeled: $W_{\text {excl }}, W_{\text {coh }}$, and $W_{\text {cons }}(x)$. Each type of interconnection is directly, related to one of the three energy terms $\left(E_{\text {excl }}, E_{c o h}\right.$, $\left.E_{\text {cons }}(x)\right)$ described in an earlier section of this proposal.

* Because in section 2 we chose to define the desired final state state of the network as a minımum of onergy. $z$ positive (penalty) weight in section 2 corresponds to an inhibition here. 


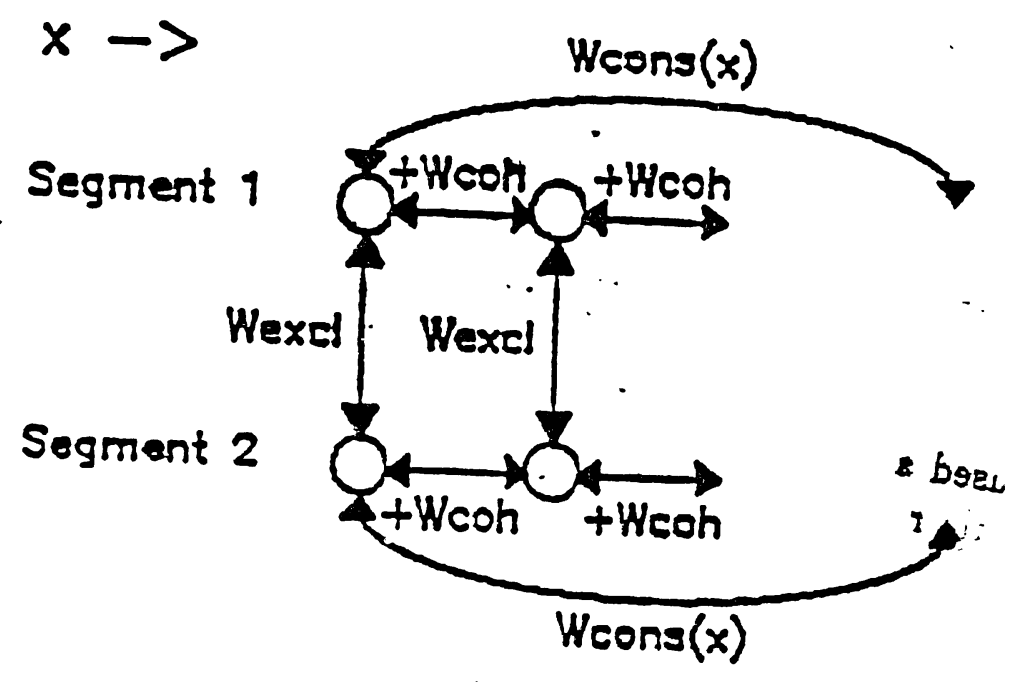

Figure A.1

Representative Section of a Neural Network Segmenter.

The $W_{\text {excl }}$ exclusion connections are columnar and inhibitory and thereby reduce the probability that a pixel will be assigned to more than one segment. The $W_{\text {coh }}$ cohesion connections are to adjacent neurons within the same segment (ie. row) and are mutually excitatory. These connections cause nearby pixels to be assigned to the same segment if no other connections (eg. the $W_{\text {cons }}(x)$ connection) cause a reversal of classifications.

The $W_{\text {cons }}(x)$ consistency connections are inhibitory and occur within the same segment. In contrast to the other two types of types of connections, the $W_{\text {cons }}$ connection is a function of $x$. This type of connection is particularly significant because it is used to discriminate between different segments. The network is driven to classify two interconnected pixels to be from different segments if the connection is strongly inhibitory. In this example network configuration, the $W_{\text {cons }}$ connection "jumps over" or spans two intervening neurons (ie. $\nu=3$ ). This type of connection is only strongly inhibitory when the input data, $f(x)$, from these intervening positions is not well modeled by a straight-line segment. Specifically, the inhibitory strength of a particular $W_{\text {cons }}$ connection is large if $f(x)$ for intervening $x$-positions cannot be well predicted from a linear interpolation using $f(x)$ measured at the two "endpoints" of the connection.

For this example of neural network simulation, we used an idealized input function which is plotted in the following illustration. The data could represent idealized measurements of luminance or depth. Directly below the graph is a diagram of 16 of the 64 neurons contained in the simulated network. The neurons are aligned directly below the corresponding data points in the graph. The results of the simulation are represented by the shading of the individual neurons. The unshaded neurons were "on" (ie $V=1$ ) at the end of the simulation. 
In this example, the neural network was simulated using the Boltzmann model ${ }^{7}$ with simulated annealing ${ }^{15}$. Initially the temperature was set very high, such that all model neurons had an essentially equal probability of being "on" or "off". The temperature was slowly lowered to zero so that an optimal or near-optimal solution could be obtained from the network. Each neuron's input-output function was the same:

$$
V=\frac{1}{1+e^{-(\operatorname{Vin}+0.5) / T}}
$$

where $\mathrm{T}$ is the temperature and

$$
V_{i n}=\sum_{i \neq j} w_{i j} V_{i}
$$

where $V_{i}$ are the outputs of the other neurons. The 0.5 constant is equivalent to an excitation bias which will drive the neuron "on" if strong inhibitory inputs are not active. $W_{\text {cons }}(x)$ is calculated from the input data. The pertinent synaptic transmission coefficients for the simulation are listed below:

$$
\begin{gathered}
\{\boldsymbol{\nu}\}=3,-3 \\
W_{\text {excl }}=-A=-1 \\
W_{\text {coh }}=+B=0.4 \\
W_{\text {cons }}(x)=-C P(x, x+3)=-.33 P(x, x+3) \\
W_{\text {cons }}(1)=0 \\
W_{\text {cons }}(2)=0 \\
W_{\text {cons }}(3)=-0.333 \\
W_{\text {cons }}(4)=-0.167 \\
W_{\text {cons }}(5)=-0.333 \\
W_{\text {cons }}(6)=0 \\
W_{\text {cons }}(x>6)=0
\end{gathered}
$$

In the future, simulations using a system of non-linear differential equations ${ }^{12}$ will extend this simulation study to more accurately model proposed VLSI implementations. 
Figure A.2

Example of Segmentation Network Simulation
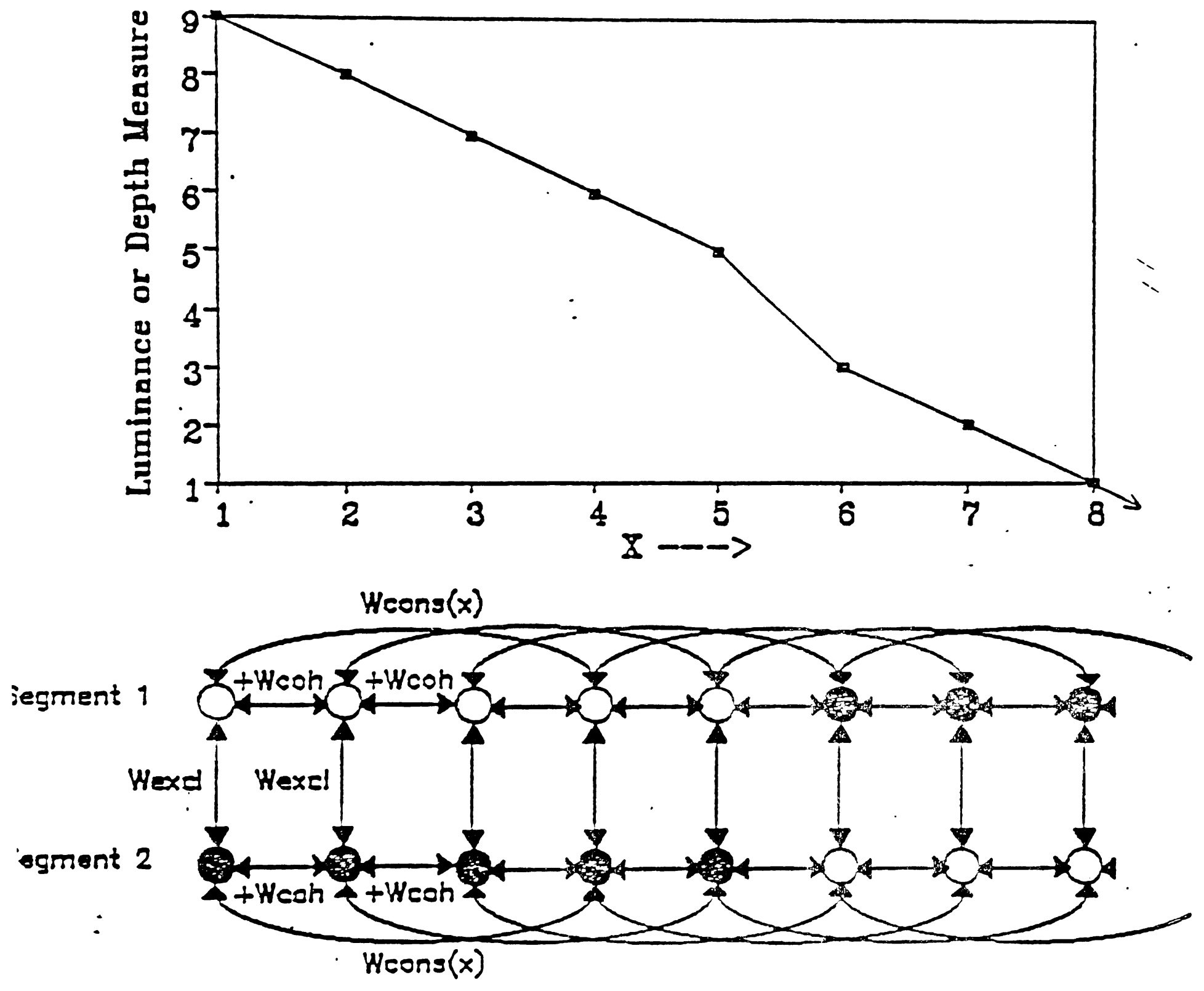


\section{References}

1. O.D. Faugeras, et al. , "Segmentation of Planar and Quadratic Patches from Range Data," CVPR, (1983).

2. D. H. Ackley, G. E. Hinton, and T. J. Sejnowski, "A Learning Algorithm for Boltzmann Machines," Cognitive Science 9 pp. 147-169 (1985).

3. P. Besl and R. Jain, "Range Image Understanding," Invited Paper, CVPR, (1985).

4. J. Canny, "Finding Edges and Lines in Images," MIT AI Laboratory Technical Report 720, (June 1983).

5. A. Cowart and W. E. Snyder, "An Iterative Approach to Region Growing Using Associative Memories," IEEE Trans PAMI, (May 1983).

6. R.O. Duda, D. Nitzan, and P. Barrett, "Use of Range and Reflectance Data to Find Planar Surface Regions," IEEE Trans. PAMI Vol. 1, No. 3 pp. 259-271 (July 1979).

7. S.E. Fahlman and G.E. Hinton, "Connectionist Architectures for Artificial Intelligence," Computer, pp. 100-109 (January, 1987).

8. S. Geman and D. Geman, "Stochastic Relaxation, Gibbs Distributions, and Bayesian Restoration of Images," IEEE Trans. PAMI Vol. 6, No. 6(Nov. 1984).

9. R.M. Haralick, "Digital Step Edges from Zero Crossing of Second Directional Derivatives," IEEE Trans. PAMI Vol. 6, No. 1 pp. 58-68 (1984).

10. M.W. Hirsch and S. Smale, Differential Equations, Dynamical Systems, and Linear Algebra, Academic Press (1974).

11. J. J. Hopfield, "Neurons with Graded Response Have Collective Computational Properties Like Those of Two-State Neurons," Proc. Natl. Acad. Sci. USA 81:30883092(1984).

12. J. J. Hopfield and D. W. Tank, "Neural Computation of Decisions in Optimization Problems," Biol. Cybern. 52:141-152(1985).

13. J. J. Hopfield and D. W. Tank, "Computing with Neural Circuits: A Model," Science 233:625-633(1986).

14. B. K. P. Horn, Robot Vision, MIT Press, Cambridge, Mass (1986).

15. S. Kirkpatrick, C. D. Gellatt, and M. D. Vecchi, "Optimization by Simulated Annealing," Science 220:671-680(1983).

16. A. Mitiche and J. K. Aggarwal, "Detection of Edges Using Range Information," IEEE Trans. PAMI Vol. 5, No. 2 pp. 174-178 (Mar 1983 ).

17. W.A. Perkins, "Area Segmentation of Images Using Edge Points," IEEE Transactions on Pattern Analysis and Machine Intelligence PAMI-2 (1) pp. 8-15 (January 1980).

18. J. Serra, Image Analysis and Mathematical Morphology, Academic Press (1982).

19. W. E. Snyder and C. D. Savage, "Content-addressable Read/Write Memories for Image Analysis." IEEE Trans on Computers C-31(Oct 1982). 